\title{
Study of Track Reconstruction using Retina algorithm for charged particles in magnetic field
}

\section{W. DENG ${ }^{1}$}

Central China Normal University \& Université Libre de Bruxelles

BD du Triomphe 151, 1050 Brussels, Belgium

E-mail: dengdandanemails. ccnu.edu.cn

\author{
G. DE LENTDECKER ${ }^{\mathrm{b}}$, G. HUANG $^{\mathrm{a}}$, F. ROBERT ${ }^{\mathrm{b}}$, Z. SONG ${ }^{\mathrm{a}, \mathrm{b}}$, D. WANG ${ }^{\mathrm{a}}, \mathrm{Y}$. \\ YANG ${ }^{\mathrm{b}}$ \\ ${ }^{a}$ Central China Normal University, \\ NO.152 Luoyu Road, Wuhan, 430079 Hubei, P.R.China \\ ${ }^{b}$ Université Libre de Bruxelles, \\ Av. F.Roosevelt 50, 1050 Brussels, Belgium \\ E-mail: Zixuan.Song@ulb.ac.be
}

\begin{abstract}
Real-time track reconstruction in high energy physics experiments at colliders running at high luminosity is very challenging for trigger systems. When it is run on FPGAs, the Retina algorithm has been used in High Energy Physics experiments mainly in the case of parallel plane detection layer without magnetic field such as the LHCb VELO detector. However another interesting geometry is a tracking detector with a barrel shape made of multiple concentric cylindrical layers surrounded by a strong (several Tesla) magnetic field. In this paper we introduce our simulation work of reconstructing the charged particle trajectory in the strong magnetic field environment by using the Retina algorithm under the structure of this barrel shape detector. Based on simulations, preliminary results on the track reconstruction resolution are presented.
\end{abstract}

KEYWORDS: Track reconstruction; Pattern recognition; Retina algorithm;

Topical Workshop on Electronics for Particle Physics (TWEPP2018)

17-21 September 2018

Antwerp, Belgium

\section{${ }^{1}$ W.DENG}

Supported by the National Key Research and Development Program of China under Grant No. 2016YFE0100900 


\section{Introduction to the Retina Algorithm in particle track reconstruction}

In High Energy Particle (HEP) physics experiments, the track reconstruction is always one of the important steps to identify the particles. Over the last decades, as the collider luminosity keeps increasing and therefore the rate of particles to be detected by the equipment, more rapid and efficiency triggering system have been required [1][2]. Therefore, new pattern recognition algorithms have been recently introduced in HEP. Retina is one of them.

The Retina algorithm is a recognition algorithm that finds the expected trajectory by scanning a discrete multi-dimension parameter space where each bin, also called cell, represents a pattern or a particle trajectory and by computing, for each cell, the "distance" between the detected hits and the corresponding pattern [3][4]. In our previous studies [5], the Retina algorithm has been applied to the reconstruction of linear particle trajectories in parallel-layer detector structures without magnetic fields. The initial position and direction of the straight line track are estimated by the Retina algorithm. Two firmware implementations have been developed on FPGA and tested with a Xilinx KC705 evaluation board [6].

In the present paper, we focus our study on the use of the Retina algorithm to reconstruct curved tracks from charged particles passing through a magnetic field. The goal of the track reconstruction in this case is to measure the transverse momentum, $\mathrm{P}_{\mathrm{t}}$, of the charged particles and their initial direction angle. While the ultimate goal is to perform the track reconstruction of bended trajectories with the Retina algorithm on FPGA devices, we present here our preliminary studies performed with simulations.

\section{Track Simulation and reconstruction with Retina in magnetic field}

The first part of this section presents the detector geometry and the simulation of charged particles inside. In the second part we describe the application of the Retina algorithm to reconstructed the curved particle tracks.

\section{1 detector geometry setup \& track generation}

In order to investigate the feasibility and performance of the Retina trajectory reconstruction for a barrel-shape multi-layer tracker in a strong magnetic field, we first developed a simple simulation and then used a more complete simulation based on GEANT4 to better simulate the detector effects.

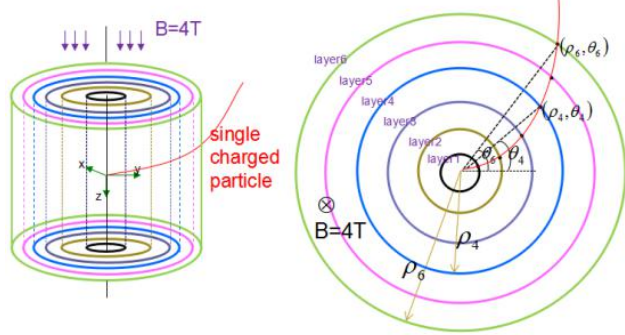

Figure 1. Detector geometry and track of charged particles in ideal case.
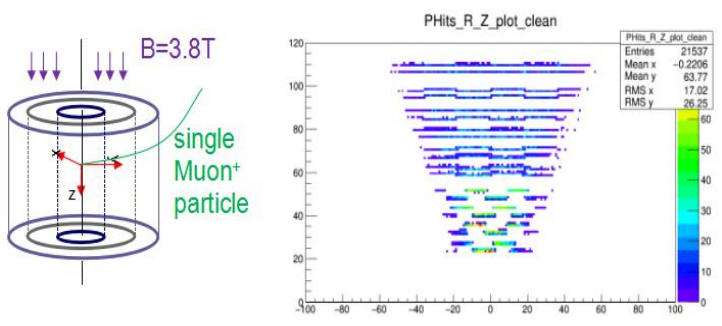

Figure 2. Detector geometry and track of charged particles generated by GEANT4.

Our simple simulation consists in an ideal detector model with a mathematical modeling of the charged particle trajectory. Figure 1 shows the detector geometry and the environmental configuration in this ideal case, with a six layer barrel tracking detector surrounded by a $4 \mathrm{~T}$ magnetic field along the Z-axis. The concentric cylindrical layers are from 0.2 meters 
(innermost 1st layer) to 1.15 meters (outermost 6th layer) along the radius. One event consists in a single charged particle starting at the center $(0,0,0)$ of these cylinder layers with a random initial angle, and crossing six barrel layers from inner to outer, providing the corresponding position information at each layer. This position information (coordinates) is used as the input of the Retina algorithm to reconstruct the Pt and initial flight direction of the particle. In presence of a magnetic field, the charged particle trajectories will bend. The trajectory changes from a straight line to a partial arc. We assume that the magnetic field strength (B) is equal to 4 Tesla and the particle is a muon of positive charge $(\mathrm{Q})$. The relationship between the radius of the $\operatorname{arc}\left(\mathrm{r}_{0}\right)$ and the particle transverse momentum $\left(\mathrm{P}_{\mathrm{t}}\right)$ is given by eq.(1) while the relationship between the hit position at each layer and the particle $P_{t}$ is given by eq.(2), where $\theta_{0}$ represents the initial direction angle of the particles and $\theta_{\mathrm{n}} \rho_{\mathrm{n}}$ represent the particle coordinate in the nth layer.

$$
r_{0}=\frac{P_{t}}{0.3 B Q}=\frac{P_{t}}{1.2} \ldots \ldots . .(1) \quad \sin \left(\theta_{\mathrm{n}}-\theta_{0}\right)=\frac{\rho_{\mathrm{n}}}{2 r_{0}} \ldots \ldots \ldots(2)
$$

After this ideal detector simulation we have then implemented a more realistic simulation based on the GEANT4 software [7]. In this case, the detector geometry and environmental configuration are similar to the above ideal case (see Figure. 2). All muon particles will start at point $(0,0,0)$ and go across 10 barrel-like layers. The right side of the Figure 2 shows the distribution of the hits accumulated on the 10 layers from 20000 tracks in the R-Z plane. From the graph, the detailed structure this more realistic barrel tracker is clearly visible.

By default, the effects of Multiple Scattering and detector resolution are included when we generate tracks with GEANT4. Therefore to compare the results between GEANT4 and our simple model, we have also added both detector effects in the track generation step of our ideal case. The position resolution of the detector we set in the ideal case amounts to $100 \mathrm{um}$ and the thickness of detector layer corresponds to $10 \%$ of the radiation length of the detector material.

\subsection{Application of the Retina Algorithm for curved particle tracks}

There are two main steps to apply Retina algorithm to reconstruct curved tracks in magnetic field (see figure 3): the first step is to linearize the patterns. For that we choose the polar coordinate system $(\rho, \theta)$ to represent the position information. We assume that the particles with high Pt will only slightly bend when they go through the magnetic field, so the change of direction $\theta_{\mathrm{n}}-\theta_{0}$ is almost equal to 0 and $\sin \left(\theta_{\mathrm{n}}-\theta_{0}\right)$ is almost equal to $\theta_{\mathrm{n}}-\theta_{0}$. Consequently the exact equation (1) (see Figure 3) simplifies and becomes the linear equation (2). Naturally, the Retina parameter space becomes the $(\rho, \theta)$ plane as the equivalent $\left(1 / \mathrm{P}_{\mathrm{t}}, \theta_{0}\right)$.
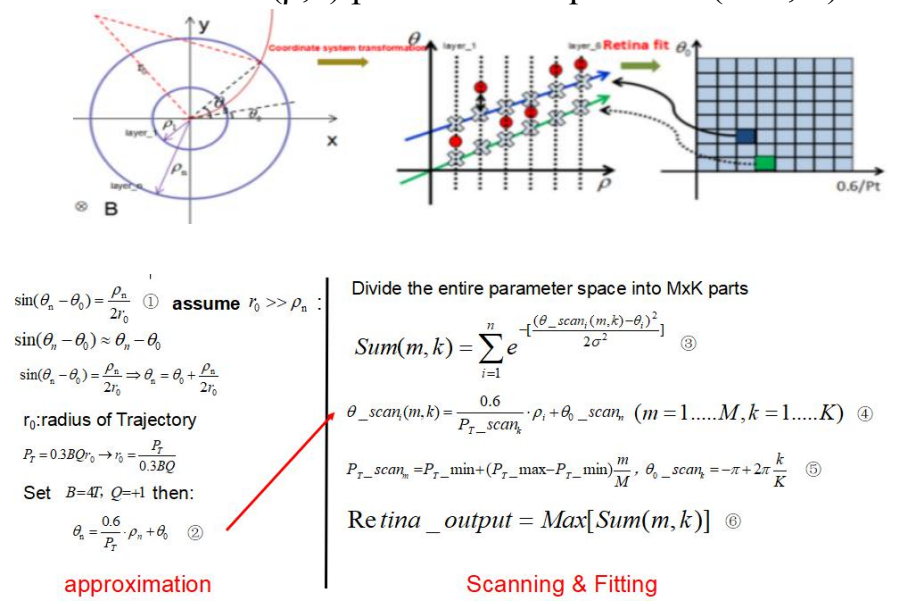

Figure 3. Steps to reconstruct curved tracks in a magnetic field with Retina algorithm 
The second step is "Scanning \& Fitting". The full parameter space is divided into several cells: $\mathrm{M}$ bins for $1 / \mathrm{P}_{\mathrm{t}}$ and $\mathrm{K}$ bins for the initial angle $\theta_{0}$. Then, for each $(\mathrm{m}, \mathrm{k})$ cell, formula (3) in Figure 3 is used to accumulate the "relevance" between the expected hit position $\left(\rho_{\mathrm{i}}, \theta \_\operatorname{scan}_{\mathrm{i}}\right)$ and the measured hit position $\left(\rho_{\mathrm{i}}, \theta_{\mathrm{i}}\right)$. Equation (4) and (5) represent the position calculation of the expected hit for a certain $(\mathrm{m}, \mathrm{k})$ cell of the parameter space. At last, we scan the whole parameter space to find the maximum value of the Sum (3) and output the corresponding $\mathrm{P}_{\mathrm{t}} \mathrm{scan}_{\mathrm{m}}$ and $\theta$ ${ }_{0}$ scan $_{\mathrm{k}}$ as the reconstructed result of $\mathrm{P}_{\mathrm{t}}$ and of the initial angle $\theta_{0}$ of the particle (see formula (6) in Figure 3). At this point, the entire process of trajectory reconstruction using Retina algorithm is completed.

In our simulations, the range of $\mathrm{P}_{\mathrm{t}}$ is from $1 \mathrm{GeV} / \mathrm{c}$ to $50 \mathrm{GeV} / \mathrm{c}$ and the range of $\theta_{0}$ is from $-\pi$ to $\pi$. We tried two binning granularity: either 200 bins for $P_{t}$ times 400 bins for $\theta_{0}$ or 500 bins for $\mathrm{P}_{\mathrm{t}}$ times 1000 bins for $\theta_{0}$. Note that the parameter $\sigma$ from formula (3) represents the weight adjustment parameter in the Retina algorithm. We assume all the positions have the same weight for the track reconstruction, so we fix $\sigma$ to 1 in our simulation.

\section{Performance and results}
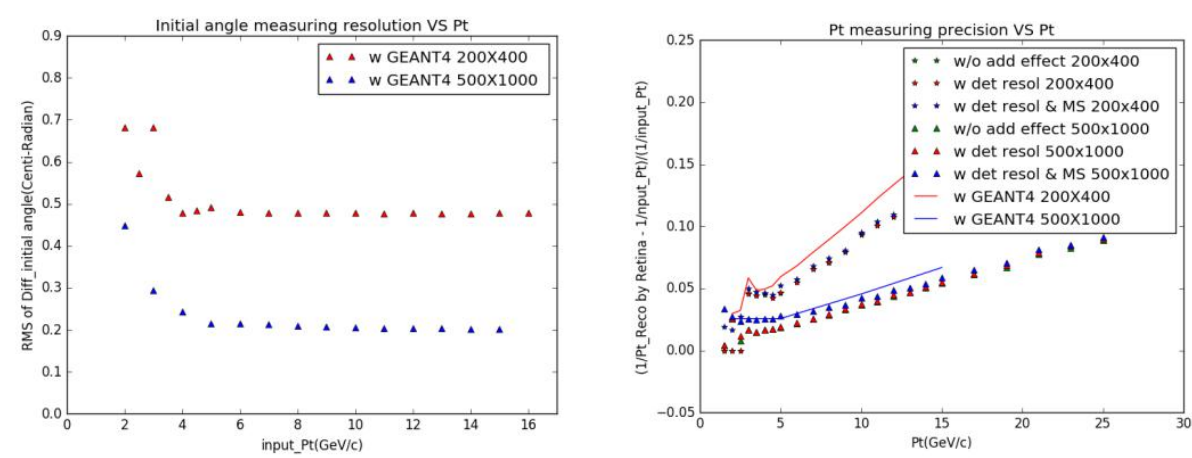

Figure 4. Pt and initial angle performance reconstructed by Retina algorithm.

The Figure 4 shows the performance of the reconstruction by Retina for tracks generated as described in chapter 2 . The left side of Figure 4 shows the resolution on the initial angle $\theta_{0}$ as a function of the generated particle $\mathrm{P}_{\mathrm{t}}$ for the two GEANT4 parameter space granularities. The right side of Figure 4 shows the $P_{t}$ resolution as a function of the generated particle $P_{t}$ for both parameter space granularities and for the simple model as well as for the GEANT4 model. For the simple model, simulations with and without any detector effects are also shown.

From Figure 4 (right) we can see that the $P_{t}$ resolution is quite close between the simple model and the GEANT4 model. Also, as expected, because of the bending, the $\mathrm{P}_{\mathrm{t}}$ resolution degrades with high $\mathrm{P}_{\mathrm{t}}$. Note also that at very low $\mathrm{P}_{\mathrm{t}}$, less than $2.5 \mathrm{GeV} / \mathrm{c}$, the resolution degrades because the linear approximation is not valid anymore. Finally we can also observe that the high Pt resolution improves by a factor $\sim 2$ when we increase the parameter space granularity.

Besides, from Figure 4 we can find that there is an abrupt jump at $3 \mathrm{GeV} / \mathrm{c}$ under the condition of the $200 * 400$ space granularities. This is cased by two factors: One is the linear approximation we used began to become more different from the actual trajectory at $3 \mathrm{GeV} / \mathrm{c}$, the distribution of the different between initial angle reconstruction by the Retina algorithm and generated by simulator are no longer Gauss shape. Another is the space granularities we set is 
not high enough, we can find from result (Figure 4) that the abrupt jump disappeared when we increasing the scanning bins from 200 times 400 to 500 times 1000 .

\section{Discussion and Conclusion}

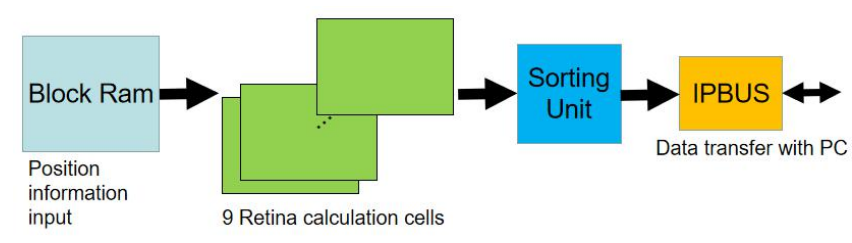

Figure 5. Structure of the Retina algorithm firmware design.

In this note, our research is targeted to adapt Retina algorithm to a tracker detector with cylindrical geometry and complex physical environment. Due to the magnetic field effects, charged particle trajectories are bent and treated as partial arc. A first Retina modelling of track reconstruction under the situation of particles in a magnetic field with barrel-like and multilayer tracker has been built. We have shown the very preliminary results on track parameter resolution. In the further we will optimize this Retina simulation as well as start to convert it to FPGA firmware. Many generally functions have to be added to have a realistic detector simulations and we also have to check the resolution of the algorithm as measure of many tracks in the same time.

By the way, we are already working on the implementation of the Retina algorithm in FPGA. Figure 5 shows the current structure of the Retina algorithm firmware design. The position information of the hits for each track will be stored in BRAM and be read out by the Retina calculation cells. After calculation and fitting the results information will be send to PC via the Communication module IPBUS[8]. We plan to mainly use the DPS48 and BRAM resources in FPGA to implement Retina calculation cells. After the work is done,we will then compare the hardware performance of our design to our simulation results as well as other fast track trigger algorithm.

\section{References}

[1] CMS Collaboration, The CMS trigger system, 2017 JINST 12 P01020. Doi: 10.1088/1748-0221/12/01/P01020.

[2] F. Palla et al., Track Finding in CMS for the Level-1 Trigger at the HL-LHC, 2016 JINST 11 C03011.

[3] R. Cenci, et al., First Results of an “Artificial Retina” Processor Prototype, EPJ Web Conf. 217 (2016) 00005. Doi: 10.1051/epjconf/201612700005.

[4] Alessio Piucci, Reconstruction of tracks in real time in the high luminosity environment at LHC, Master thesis, Universit`a degli Studi di Pisa (2013-2014).

[5] Z. Song, et al., Study of hardware implementation of fast tracking algorithms, 2017 JINST 12 C02068.

[6] Xilinx UG810. KC705 Evaluation Board for the Kintex-7 FPGA User Guide. https://www.xilinx.com/support/documentation/boards_and_kits/kc705/ug810_KC705_Eval_Bd.pdf

[7] S. Agostinelli, "Geant4: A simulation toolkit", Nucl. Instrum. Meth. A, vol. 506, no. 3, pp. 250-303, 2003.

[8] Larrea C G , Harder K, Newbold D, et al. IPbus: a flexible Ethernet-based control system for xTCA hardware. Journal of Instrumentation, 2015, 10(2):C02019-C02019. 